\begin{tabular}{|llll}
\hline International Journal of Applied & $\mathbf{A}$ & $\mathbf{I}$ \\
\hline
\end{tabular}

Review Article

\title{
Accuracy of the Positioning Systems for the Tracking of Alzheimer's Patients - A Review
}

\author{
Omid Toutian Esfahani ${ }^{1}$, Ata Jahangir Moshayedi ${ }^{1,2, *}$
}

(1) Control engineering group, Islamic Azad University (Khomeini shahr branch), Esfahan, Iran.

(2) Department of Electronic Science, University of Pune, Pune: 411007, India.

\section{Article history:}

Received 26 July 2014

Reviewed 01 September 2014

Revised 31 October 2014

Accepted 08 November 2014

Published 22 November 2014
Abstract. Robotic revolution made the position monitoring systems become more and more demanding in the industry. Knowing the position of an object will help for the better navigation as well as control of systems. This review is about the current position monitoring systems with an overlook of their applications in helping Alzheimer's patients. Here we have included the indoor and outdoor methods and the comparison between the common methods used in position monitoring systems. A list of various methods used for the position monitoring has been included in the conclusion section based on their accuracy and cost.

Keywords: positioning systems, Alzheimer, GPS, ultrasonic, RSSI, RFID.

\section{Introduction}

Alzheimer's disease is a type of dementia that cause a problem for memory, thinking and behavior. It starts slowly and become worse and over the time become strong enough to affect daily activities. In 2011, more than 24 million people were experienced Alzheimer disease all around the world and it is predicted that this number will be doubled every 20 years ${ }^{[1]}$.

Recently, several strategies developed for decreasing the disease progression. Among these strategies, targeting the clearance of amyloid-B peptide (which is an important factor in disease development) are most important and advanced ${ }^{[2]}$. Some strategies are also developed to predict Alzheimer's disease in people with 50 to $70 \%$ chance to develop dementia within the next 5 to 7 years $^{[3]}$.

One of the biggest problems with Alzheimer's patients is that they can easily get lost ${ }^{[1]}$, especially when they are out of home and alone. Therefore, this is very important for the care-giver to keep track of the patient's location in an indoor and outdoor environment using suitable positioning systems. A positioning system is categorized into indoor and outdoor.

The positioning system is a mechanism for specifying the location of desired objects or people in different environments. The technologies for this task are ranging from a worldwide coverage with meter accuracy to a workspace coverage with sub-millimeter accuracy ${ }^{[4]}$.

The main aim of this review is to list the various posi-

*Corresponding author: A. J. Moshayedi

๘: +912025699841

凶: moshayedi@electronics.unipune.ernet.in tion monitoring systems that can be used for Alzheimer's patients. Based on the reported work, the positioning systems are divided into indoor and outdoor. In this review, initially some indoor and outdoor positioning systems were surveyed and compared with each other, including infrared, ultrasonic, received signal strength indication (RSSI), Global Positioning System (GPS), etc.

In the next sections, a general overview of some positioning systems developed for Alzheimer's patients is given. And finally, the review concludes with a table that helps readers to compare different positioning systems with each other.

\section{Positioning Systems}

As it was mentioned before the position monitoring systems are divided into the indoor and the outdoor. Both methods are described follows.

\subsection{Indoor Positioning Systems}

An indoor positioning system (IPS) is a net of devices used to wirelessly locate objects or people within the construction $^{[5]}$. Instead of using satellites, an IPS (most of the times) is based on magnetic positioning, dead reckoning, or nearby anchors (nodes with a known position), which either actively pinpoint tags or make available ambient location or environmental context for devices to get sensed ${ }^{[6]}$. The current indoor positing methods is summarized in Fig. 1.

\subsubsection{Infrared Positioning Systems}

The first indoor positioning system is called "active badges". The system is based on infrared beacons which is shown in Fig. 2. These beacons transmit a unique code 


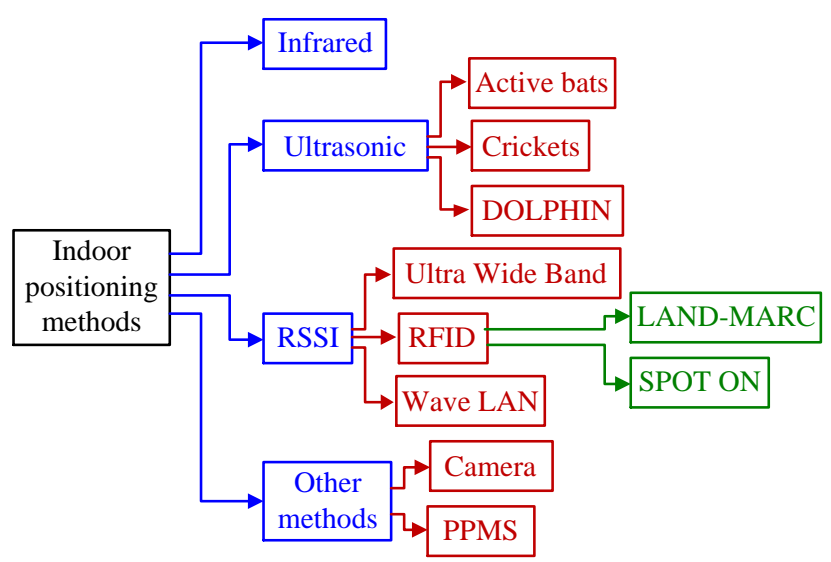

Fig. 1. indoor positioning systems

every 15 minutes. In this method the whole building should be covered with infrared (IR) sensors. These sensors detect the transmissions and identify the location of the carrier ${ }^{[7]}$. Then all the informations, collected from sensors, are sent to a central server for processing. The accuracy is about $7 \mathrm{~cm}$ for this system.

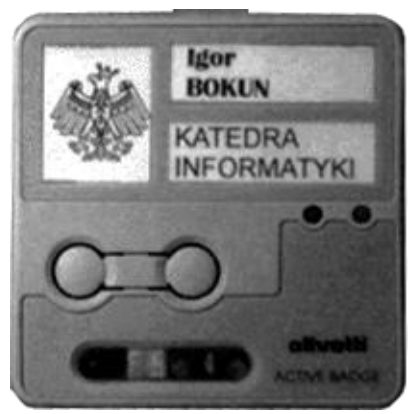

Fig. 2. Active badges ${ }^{[8]}$

\subsubsection{Ultrasonic Positioning Systems}

The ultrasonic positioning systems are divided into three main systems of

a. Active bats,

b. Crickets,

c. and Dolphin.

Active bats users are tagged with ultrasonic tags called "bats". These bats send ultrasonic signals periodically to receivers placed across the ceiling. The problem of using such system is that it takes a heavy number of sensors across the roof and their position is rather tender. The accuracy of this system is about $9 \mathrm{~cm}^{[9]}$.

Crickets which is shown in Fig. 3 is the small ultrasonic device developed by Massachusetts Institute of Technology (MIT). Cricket uses a combination of radio frequency $(\mathrm{RF})$ and ultrasound technology for obtaining the location information of the host devices. The system consists of two main parts,

- the beacons which sent data through RF channel,

- and listeners which listen for signals.

With each RF advertisement, the beacon transmits a concurrent ultrasonic pulse. Listeners listen for RF signals, and when they receive the first few bits of RF signal, continue to listen for the corresponding ultrasonic pulse. When this pulse arrives, the listener calculates the distance from the beacon by taking advantage of the differ- ence in propagation speeds between RF (speed of light) and ultrasound (speed of sound) signals ${ }^{[10]}$. The advantage of this system is that the system is cost effective and the accuracy is about $2 \mathrm{~cm}$ which is a good result in comparison with other systems.
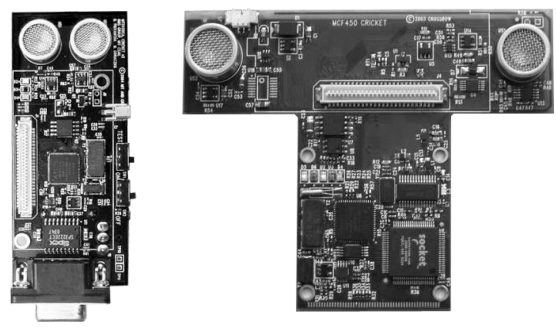

Fig. 3. Cricket beacons and listeners ${ }^{[11]}$

DOLPHIN (Distributed Object Locating System For Physical Space Inter Networking) is a system which uses wireless sensor nodes to determine the indoor location of an object or a person ${ }^{[12,13]}$. Nodes send RF and ultrasonic signals, also use a distributed positioning algorithm to locate objects and persons. This algorithm enables DOLPHIN to work with minimum manual configuration. The system accuracy is about $2 \mathrm{~cm}$ in indoor environments.

\subsubsection{RSSI Positioning Systems}

The RSSI method as the broad form of received signal strength indication which is acting based on principle of measurement of the power level received by sensors. In this method distance can be approximated based on the relationship between the transmitted and the received signal intensity.

The RSSI usually uses RF signals ${ }^{[14]}$. Primarily, a test run should be accomplished to determine the test database for different sensors. The RSSI data received by the sensor; then, compared to the test database; and the location is determined by matching the collected data by sensors and data of the database. The wall and obstacle has a remarkable effect on the receiver's signal accuracy as the drawback of the system ${ }^{[15]}$.

RSSI systems based on RF signals are divided into three main systems ${ }^{[16]}$ of,

a. Wave LAN,

b. Ultra Wide Band,

c. and RFID technology.

Wave LAN system uses signal strength and signal to noise ratio of wave LAN wireless network interface cards (NIC) ${ }^{[17]}$. The advantage of this system is using the general wireless networking infrastructure of the building. However, the tracked object must carry a NIC. The accuracy of this system is about 3 meters.

Ultra Wide Band (UWB) system uses Time of Arrivals (TOA) to find the location of an object or a person in indoor environments which enable these organizations to deliver a degraded performance as it expressed in Fig. 4.

As Fig. 4 shows, the emitter sends a signal and it is received by four receivers which are placed in known locations. The clock network is applied to supply a reference clock for all recipients and data network is utilized to transmit data between receivers and PC. The data received to the computer are used to determine the position of the emitter using a TOA technique. This system's error 


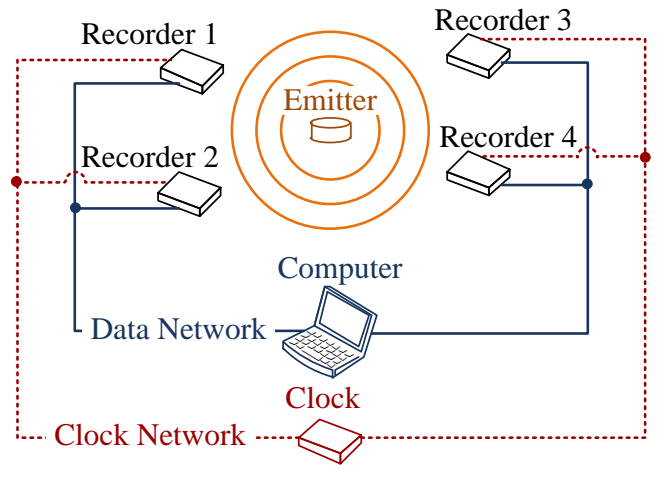

Fig. 4. Indoor positioning system using UWB

reported below $30 \%$ for $95 \%$ of all locations determined and the accuracy is $10 \mathrm{~cm}^{[18]}$.

$\boldsymbol{R F I D}$ (radio frequency identification) systems consist of small tags, attached to objects and RFID readers. When these tags are identified by RFID the readers, tags send some unique identifying information to the reader, which facilitates the reader to locate tags and extract some information from them. Some tags are powered by and work at short ranges. Others use local power sources like batteries or have no battery, but receive energy from electromagnetic fields and work at hundreds of meters. One of the greatest advantages of RFID systems is that they are cost effective ${ }^{[19]}$. Two most popular systems using RFID are LAND-MARC and SPOT ON.

i. LAND-MARC system exists of two main parts which are RFID active tags and Readers. It is shown in Fig. 5. In these systems the RFID active tag is preprogrammed with an ID to help the readers to identify it. The RFID readers has eight power levels, which determine the distance between the active tag and the reader. Number 1 is the shortest range and number 8 is the longest. The accuracy is between 1 to $2 \mathrm{~m}$ for this method ${ }^{[20]}$.

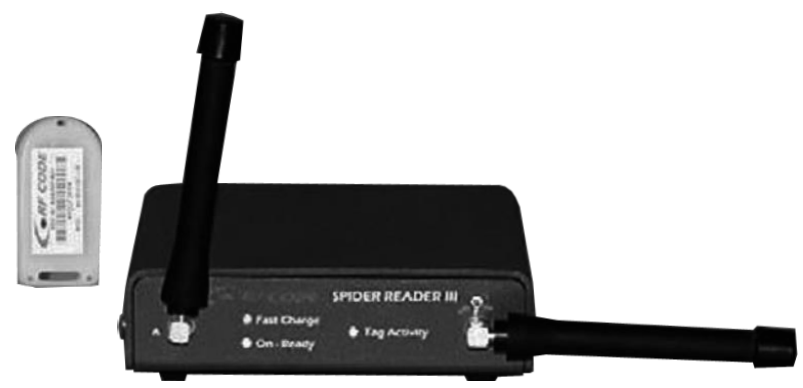

Fig. 5. RFID tag and reader ${ }^{[20]}$

ii. SPOT ON method is the system for the new tagging technology for three dimensional (3D) location detection based on received signal strength (RSS). The SPOT ON system is shown in Fig. 6. This system uses an embedded hardware system named Hydra Micro server, which has both Ethernet and RS232 ports can be used for INTERNET working. In these systems the base stations are utilized to provide RSS measurements and also send these data to the host. The server processes RSS values and determines the position of the object. The system accuracy is about $3 \mathrm{~m}$ which is below average compared to other methods ${ }^{[21]}$.

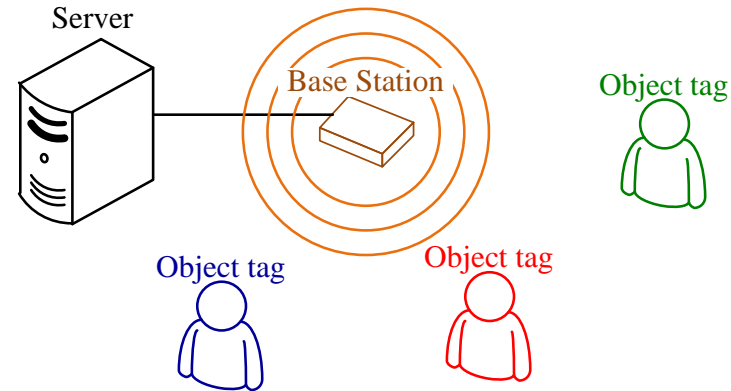

Fig. 6. SPOT ON architecture

\subsubsection{Other Methods}

The cameras can be used for indoor positioning systems. These systems have a good accuracy $(10 \mathrm{~cm})$, but have several disadvantages. As the system notable disadvantage, uses of several cameras to pass over the whole building and system cost it can be observed ${ }^{[22]}$. Another approach is the path and position monitoring system (PPMS), based on the computer mouse ${ }^{[16]}$. The computer mouse works same on the encoder wheels and interprets the cursor motion on graphical user interface (GUI), based on the light passing between attached disk and infrared sensors when the roller moves. The encoder signals are converted into motion of the mouse along $\mathrm{X}$ and $\mathrm{Y}$ axes, on the screen. Considering these features, the same principle was used for the position monitoring system. Moving the mouse ball in any direction, generates the signal which is transferred to the personal computer via an $\mathrm{RF}$ module. In order to obtain $(\mathrm{X}, \mathrm{Y})$ position as well as time and velocity, an electrical relay is switched on every second with the help of the accurate timing program running on the ATMEGA 32 microcontroller. This acts as a mouse click in every second to get the robot position. Two main limitations of this method reported which are occurring by the systematic error in computer mouse. The system is not able to monitor the turning below $5^{\circ}$; and in the case of surface roughness, sometimes the ball movement is not smooth leading to errors. The difficulty can be rectified by the shock absorber or using the optical mouse for darker backgrounds.

\section{Outdoor Positioning Systems}

Outdoor positioning systems usually use global positioning system (GPS) modules as the main part alongside with a micro controller for processing and a global system for mobile (GSM) module to communicate with control and monitoring units. The GPS method uses 24 satellites to locate the position of the desired object or person which is shown in Fig. $7^{[23]}$. GPS needs at least three satellite signals to determine the two dimensional (2D) position and more than three satellites to determine 3D position $^{[24]}$.

\subsection{GPS Related Systems}

As it was mentioned, GPS is a satellite-based navigation system which consists of 24 satellites. These satellites are located in orbit and provide the essential location based information to anyone with a GPS receiver ${ }^{[23]}$.

GPS is able to work in any weather and anywhere in the world ${ }^{[26]}$. GPS must be locked on to the signals of at least three satellites to approximation $2 \mathrm{D}$ positions and 


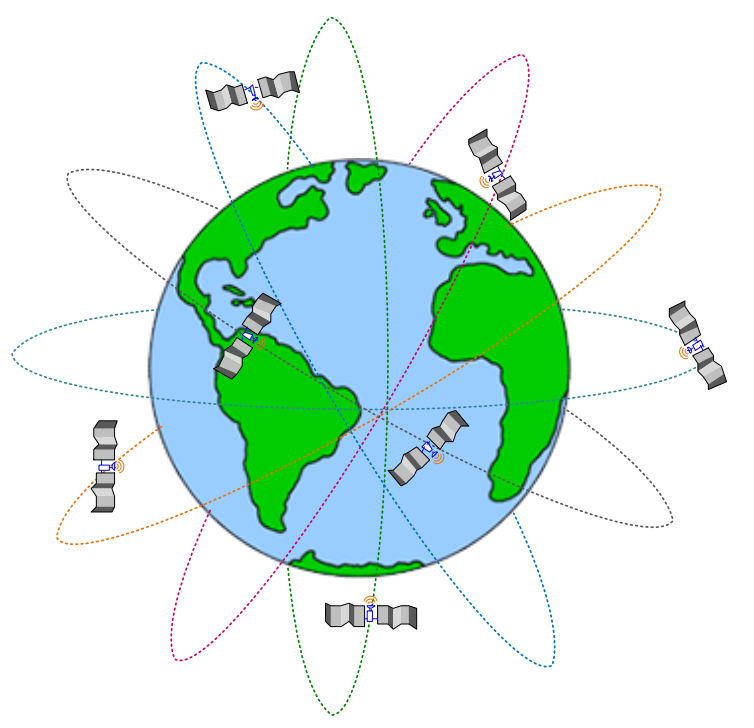

Fig. 7. GPS satellites ${ }^{[25]}$

with four or more it is able to define a user's 3D position ${ }^{[23]}$. GPS systems are not generally suitable for indoor positioning. This is because of that signals weaken by the materials used in buildings, walls and other objects $^{[27]}$. However, GPS receivers can be more potent and sensitive and some of high sensitive receivers which will be able to receive signals in an indoor environment and determine 2D and 3D position ${ }^{[28]}$. Previously, the disadvantage of the GPS was their limitation in indoor positioning ${ }^{[27]}$, but nowadays, GPS receivers are able to receive signals in indoor environments ${ }^{[29,30]}$. GPS positioning accuracy is about 1 to 5 meters ${ }^{[31]}$.

\subsection{LaMarca et al.'s Method}

Another outdoor positioning method is a place lab location system developed by LaMarca et al. in 2005, which were allowing users to determine their position in the urban environment ${ }^{[4,32]}$. This system uses RSSI of Wi-Fi hotspots and GSM broadcast towers to determine user's position. This system uses a database of known Wi-Fi hotspots and GSM broadcast towers. The accuracy is less than the GPS, with 20 to 25 meters using Wi-Fi hotspots and 100 to 150 meters for GSM broadcast towers.

\section{Positioning Systems Developed for Alzheimer's Patients}

Most of the mentioned systems represented in the previous sections can be used to track an Alzheimer's patient. However, the two most important deciding factors are the accuracy and the price that limit the position method usages. Therefore, engineers and developers should efficiently use a system which can balance these factors. As it was reported ${ }^{[28]}$, the ultrasonic and RFID technologies are the best choice for indoor environment; and a GPS based system is suited for outdoor placement ${ }^{[33]}$. Reviewed positioning systems for Alzheimer's patients are summarized in Fig. 8.

\subsection{RFID Based Methods}

Recently, an integrated system using GPS, GSM and RFID is presented ${ }^{[34]}$. The RFID systems are used to position the of patient in indoor environments and can automatically detect; if the patient leaves certain specific

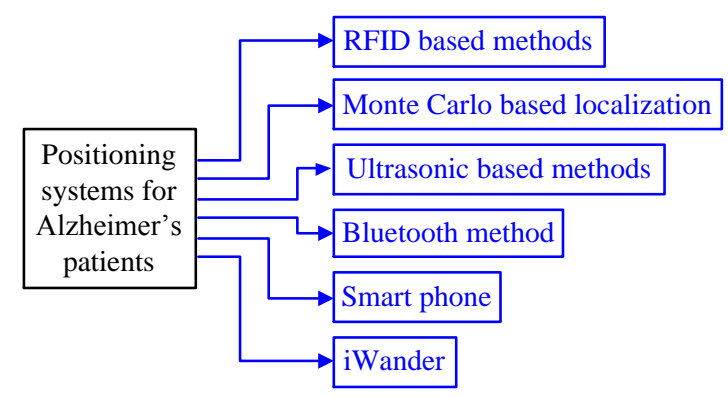

Fig. 8. Positioning systems for Alzheimer's patients

areas such as kitchen, bedroom, etc. If the patient leaves home without notice, the system sends a short message service (SMS) to caregivers and informs them. The system has a rescue mode, which can be used in an emergency situation. The system transmits an alert message to caregivers, after patient press the emergency rescue button. Another benefit of this system is that the caregivers can access the call center through the network and provide the authentication ID; and system automatically reports to the coordinates of the patient in a real-time ${ }^{[26]}$. The block diagram of this system is shown in Fig. 9.

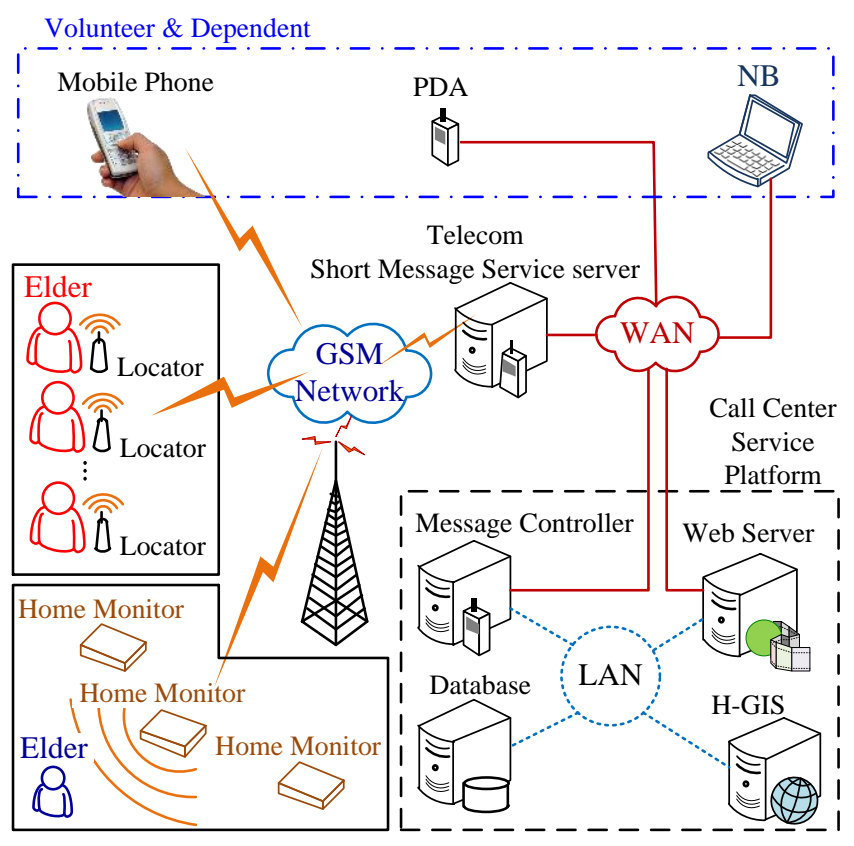

Fig. 9. Call center service platform ${ }^{[26]}$

\subsection{Monte Carlo Based Localization}

Another approach is using Monte Carlo based localization algorithm alongside with an indoor map information and static node positions to locate the person's location in indoor environments ${ }^{[34,35]}$. This system uses an indoor localized network, which consists of a base node, mobile nodes and static nodes, which are shown in Fig. $10^{[36]}$.

This system uses dynamic position tracking model (DPTM) to track the location of the mobile node in real-time. First, the system determines which static nodes are in proximity to the mobile node. After that, it finds out the motion status using built-in mobile node's accelerometer sensor and calculates the number of steps taken. Then, it extracts the heading of the mobile node using static node's proximity and mobile node's heading sensor. Prediction algorithm using particle 


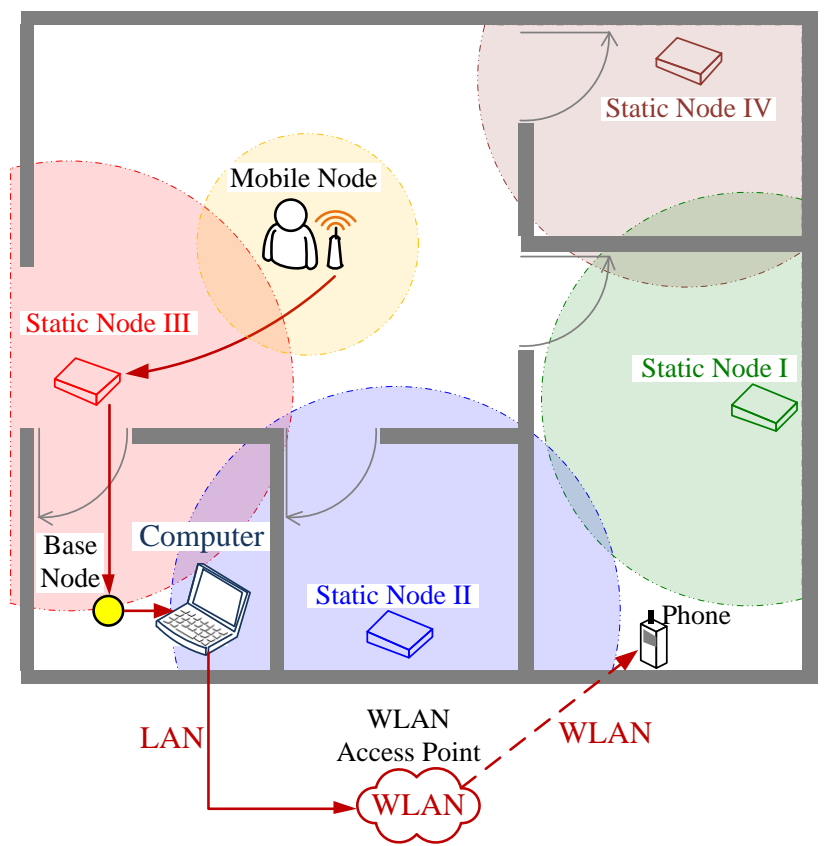

Fig. 10. Indoor localization network ${ }^{[36]}$

filtering, determines the likely position of the mobile node. Error of this system is about 1 to 3.5 meters in a health care clinic environment with 10000 meters distance covered and 7000 footsteps taken.

\subsection{Bluetooth Method}

Another cost efficient method is using Bluetooth for an indoor positioning system ${ }^{[37]}$. This system is using multiple Bluetooth beacons, all around the building, to cover all of the building area. One disadvantage of this system is that it takes about 20 seconds to detect Bluetooth beacons. This system has lower energy consumption than Wi-Fi networks, and this system is able to detect patient's activities. For Alzheimer's patients showing "wandering" behavior combined with excessive activity falls can be dangerous. Hence, a reliable fall detection system is a key feature in an assistance system for dementia and Alzheimer patients. Therefore, this system uses plaster sensor which is equipped with both three-axises digital accelerometer and three-axises digital gyroscope to have a better accuracy to detect falls.

\subsection{Ultrasonic Based Method}

A system using ultrasonic was described ${ }^{[38]}$. This system can track an ultrasonic 3D tag which in this case, attached to a wheelchair in a nursing environment. The ultrasonic emitters and receivers are deployed in the ceiling. The block diagram is depicted in Fig. 10. There are several predefined areas in the building. When the wheelchair goes into one of the fields, the subsystem notifies caregivers immediately.

\subsection{Smart Phones}

Using smart phones in humans' daily life has lots of eligible gains. A new approach for a positioning system is using smart phones' built-in GPS module to determine location of desired person ${ }^{[38,39]}$ or in this case, patient with Alzheimer disease. An example is a cloud based positioning system which is used by Microsoft Windows mobile devices ${ }^{[40-42]}$. The GPS API enables the software to communicate with built-in GPS module ${ }^{[43,44]}$. Additionally, the system calculates the distance between the patient and the central point using a sine formula ${ }^{[35]}$. If patient breach the border, then system sends an SMS to caregivers in every minutes. Each message includes the longitude and the latitude of the patient as well as his or her speed and the battery charge level.

\section{6 iWander}

$i$ Wander ${ }^{[29]}$ is an Android based application that can run on most of android devices ${ }^{[45-50]}$. This application also uses the device's built-in GPS sensor to determine the coordinates of the patient. This technology is able to receive the weather's information from the INTERNET and determine the state of dementia based on information given by the user. The collected data is then evaluated to determine the probability of wandering. $i$ Wander automatically takes action for helping patient, in order to find a safe place, notify caregivers, provide the current positioning of the patient and calls the 911 emergency service $^{[45,51,52]}$. The danger of wandering can be identified by assigning the safe zone area. In this case, firstly, it is considered that the patient is in an indoor environment where the patient is secure from the potential harms of wandering. These zones are identified by monitoring the patient locations in the areas where the phone is charged for extended periods of time. Once the patient is outside of the safe zones, the probability of wandering is determined using Bayesian network techniques. A Bayesian network is a model for determining the probability of an event happening using other variables of interest, as the variables change, inference can be applied to determine the likelihood of a specific event occurring ${ }^{[53]}$.

\section{Conclusion}

In this review, current positioning systems along with their advantages and disadvantages is discussed. The positioning over the wide range of applications in the robotic and the industry. Finding applications for lost people can be also extended to provide a help to the Alzheimer's affected people. The indoor positioning systems are separated into infrared, ultrasonic and RSSI methods; and outdoor methods consist of GPS, GSM and Wi-Fi hotspots RSS. The brief comparison between the reviewed methods is listed in Table 1.

From Table 1, it is clear that with respect to the accu-

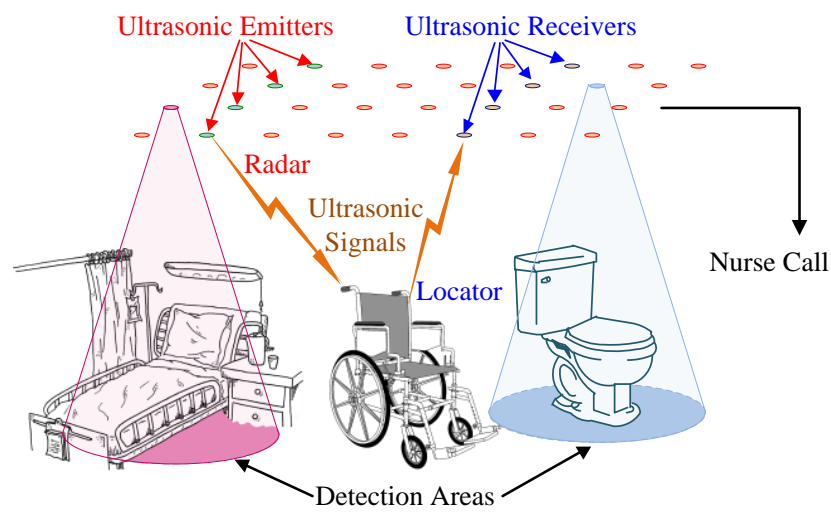

Fig. 11. Schematic diagram of the nursing care support system ${ }^{[38]}$ 
Table 1. Positioning systems' overview

\begin{tabular}{|c|c|c|c|c|}
\hline \multirow[b]{2}{*}{ System } & \multicolumn{4}{|c|}{ Features } \\
\hline & Accuracy $[\mathrm{m}]$ & Indoor & Outdoor & Cost \\
\hline GSM and Hotspot RSS ${ }^{[32]}$ & 50 & & $\checkmark$ & $\bowtie$ \\
\hline $\operatorname{GPS}^{[31]}$ & $1 \sim 5$ & $\checkmark$ & $\checkmark$ & $\bowtie$ \\
\hline Active badge ${ }^{[7]}$ & 0.07 & $\checkmark$ & & $\bowtie$ \\
\hline Active bat ${ }^{[9]}$ & 0.09 & $\checkmark$ & & $\bowtie$ \\
\hline Cricket $^{[10]}$ & 0.02 & $\checkmark$ & & $\nabla$ \\
\hline DOLPHIN $^{[13]}$ & 0.02 & $\checkmark$ & & $\bowtie$ \\
\hline WaveLAN $^{[17]}$ & 3 & $\checkmark$ & & $\bowtie$ \\
\hline $\mathrm{UWB}^{[18]}$ & 0.1 & $\checkmark$ & & $\bowtie$ \\
\hline SPOT ON $^{[21]}$ & 3 & $\checkmark$ & & $\nabla$ \\
\hline LAND-MARC $^{[20]}$ & $1 \sim 2$ & $\checkmark$ & & $\bowtie$ \\
\hline Cameras $^{[22]}$ & 0.1 & $\checkmark$ & & $\triangle$ \\
\hline
\end{tabular}

racy and the price of the ultrasonic system is the best the choice $^{[54]}$. For applications where more concern is about the accuracy, then computer vision and cameras are the desirable systems. However, the price of a computer vision system is higher than other systems and techniques. The comparisons between the mentioned methods show that the highest accuracy and cost in an indoor application is for computer vision; and for the outdoors the GPS method can be the best option.

\subsection{Future Work}

The future study will concentrate on implementing and testing the positioning system with some novel approach in terms of the algorithm and the system design for Alzheimer's patients.

\section{Acknowledgment}

Authors would like to thank Mrs. Priya Francies, the SRF of the Department of Electronic Science for the English proofreading of this manuscript.

\section{REFERENCES}

[1] C. Reitz, C. Brayne, and R. Mayeux, "Epidemiology of Alzheimer disease," Nature Reviews Neurology, vol. 7, no. 3, pp. 137-152, 2011.

[2] M. Citron, "Alzheimer's disease: strategies for disease modification,” Nature reviews Drug discovery, vol. 9, no. 5, pp. 387-398, 2010.

[3] V. Drago, C. Babiloni, D. Bartrés-Faz, A. Caroli, B. Bosch, T. Hensch, M. Didic, H.-W. Klafki, M. Pievani, J. Jovicich et al., "Disease tracking markers for Alzheimer's disease at the prodromal (MCI) stage," Journal of Alzheimer's Disease, vol. 26, pp. 159-199, 2011.

[4] H. Koyuncu and S. H. Yang, "A survey of indoor positioning and object locating systems," IJCSNS International Journal of Computer Science and Network Security, vol. 10, no. 5, pp. 121-128, 2010.

[5] K. Curran, E. Furey, T. Lunney, J. Santos, D. Woods, and A. McCaughey, "An evaluation of indoor location determination technologies," Journal of Location Based Services, vol. 5 , no. 2 , pp. $61-78,2011$.

[6] E. Furey, K. Curran, and P. Mc Kevitt, "HABITS: A history a ware based Wi-Fi in door tracking system," in In Proc. of the 9th Annual PostGraduate Symposium on The Convergence of Telecommunications, Networking and Broadcasting (PGNET-08), 2008, pp. 224-229.

[7] R. Want, A. Hopper, V. Falcao, and J. Gibbons, "The active badge location system," ACM Transactions on Information Systems (TOIS), vol. 10, no. 1, pp. 91-102, 1992.

[8] I. Bokun and K. Zielinski, "Active badges - the next generation," October 1998, [Accessed: 2014-05-30]. [Online]. Available: http://www.linuxjournal.com/article/3047

[9] M. Hazas and A. Hopper, "Broadband ultrasonic location systems for improved indoor positioning," Mobile Comput- ing, IEEE Transactions on, vol. 5, no. 5, pp. 536-547, 2006.

[10] N. B. Priyantha, "The cricket indoor location system," Ph.D. dissertation, Massachusetts Institute of Technology, 2005.

[11] "The cricket indoor location system," March 2006, [Accessed: 2014-05-30]. [Online]. Available: http: //cricket.csail.mit.edu

[12] Y. Fukuju, M. Minami, H. Morikawa, and T. Aoyama, "DOLPHIN: An autonomous indoor positioning system in ubiquitous computing environment." in WSTFEUS, 2003, pp. 53-56.

[13] M. Minami, Y. Fukuju, K. Hirasawa, S. Yokoyama, M. Mizumachi, H. Morikawa, and T. Aoyama, "DOLPHIN: a practical approach for implementing a fully distributed indoor ultrasonic positioning system," in UbiComp 2004: Ubiquitous Computing. Springer, 2004, pp. 347-365.

[14] B.-G. Lee, Y.-S. Lee, and W.-Y. Chung, "3D navigation real time RSSI-based indoor tracking application," JUCT: Journal of Ubiquitous Convergence Technology, vol. 2, no. 2, pp. 67-77, 2008.

[15] E. E. L. Lau and W. Y. Chung, "Enhanced RSSI-based real-time user location tracking system for indoor and outdoor environments," in Convergence Information Technology, 2007. International Conference on. IEEE, 2007, pp. 1213-1218.

[16] A. J. Moshayedi and D. C. Gharpure, "Development of position monitoring system for studying performance of wind tracking algorithms," in Robotics; Proceedings of ROBOTIK 2012; 7th German Conference on. VDE, 2012, pp. 1-4.

[17] P. Bahl and V. N. Padmanabhan, "User location and tracking in an in-building radio network," Technical Report MSR-TR-99-12, Microsoft Research, Tech. Rep., 1999.

[18] R. Merz, F. Chastellain, A. Blatter, C. Botteron, and P.-A. Farine, "An experimental platform for an indoor location and tracking system," in European Navigation Conference, Toulouse, France, 2008.

[19] S. A. Weis, "RFID (Radio Frequency Identification): Principles and applications," 2007.

[20] L. M. Ni, Y. Liu, Y. C. Lau, and A. P. Patil, "LANDMARC: indoor location sensing using active RFID," Wireless networks, vol. 10, no. 6, pp. 701-710, 2004.

[21] J. Hightower, R. Want, and G. Borriello, "SpotON: An indoor 3D location sensing technology based on RF signal strength," UW CSE 00-02-02, University of Washington, Department of Computer Science and Engineering, Seattle, $W A$, vol. $1,2000$.

[22] J. Krumm, S. Harris, B. Meyers, B. Brumitt, M. Hale, and S. Shafer, "Multi-camera multi-person tracking for easyliving," in Visual Surveillance, 2000. Proceedings. Third IEEE International Workshop on. IEEE, 2000, pp. 3-10.

[23] E. D. Kaplan and C. J. Hegarty, Understanding GPS: principles and applications. Artech house, 2005.

[24] M. S. Grewal, L. R. Weill, and A. P. Andrews, Global positioning systems, inertial navigation, and integration. John Wiley \& Sons, 2007.

[25] “Gps satellite constellation," September 2014, [Accessed: 2014-09-25]. [Online]. Available: http://phoneserviceslocal. com/gps-satellite-constellation/

[26] A. Fuad, M. Ridhwan, and M. Drieberg, "Remote vehicle tracking system using GSM modem and google map," in Sustainable Utilization and Development in Engineering and Technology (CSUDET), 2013 IEEE Conference on. IEEE, 2013, pp. 15-19.

[27] G. Hein, M. Paonni, V. Kropp, and A. Teuber, "GNSS indoors, fighting the fading," Part I. Marzo-Abril de, 2008.

[28] R. J. Czerniak and J. P. Reilly, Applications of GPS for surveying and other positioning needs in departments of transportation. Transportation Research Board, 1998, vol. 258.

[29] K. Ozsoy, A. Bozkurt, and I. Tekin, "Indoor positioning based on global positioning system signals," Microwave and Optical Technology Letters, vol. 55, no. 5, pp. 1091-1097, 2013.

[30] J. Hightower, A. LaMarca, and I. E. Smith, "Practical lessons from place lab," Pervasive Computing, IEEE, vol. 5, no. 3, pp. 32-39, 2006.

[31] F. Van Diggelen and C. Abraham, "Indoor GPS technology," CTIA Wireless-Agenda, Dallas, 2001.

[32] A. LaMarca, Y. Chawathe, S. Consolvo, J. Hightower, I. Smith, J. Scott, T. Sohn, J. Howard, J. Hughes, F. Potter et al., "Place lab: Device positioning using radio beacons 
in the wild," in Pervasive computing. Springer, 2005, pp. 116-133.

[33] C.-C. Lin, M.-J. Chiu, C.-C. Hsiao, R.-G. Lee, and Y.-S. Tsai, "Wireless health care service system for elderly with dementia," Information Technology in Biomedicine, IEEE Transactions on, vol. 10, no. 4, pp. 696-704, 2006.

[34] S. Lee, H. Shin, R. Ha, and H. Cha, "IEEE 802.15. 4a CSSbased mobile object locating system using sequential Monte Carlo method," Computer Communications, vol. 38, pp. 1325,2014

[35] S. TamilSelvan and M. K. Thangaraj, "Effective range-free localization scheme for wireless sensor network," International journal, 2014

[36] M. D'Souza, M. Ros, and M. Karunanithi, "An indoor localisation and motion monitoring system to determine behavioural activity in dementia afflicted patients in aged care," 2012.

[37] A. Schwarzmeier, J. Besser, R. Weigel, G. Fischer, and D. Kissinger, "A compact back-plaster sensor node for dementia and Alzheimer patient care," in Sensors Applications Symposium (SAS), 2014 IEEE. IEEE, 2014, pp. 7578.

[38] T. Hori, Y. Nishida, and S. Murakami, "Pervasive sensor system for evidence-based nursing care support," in Robotics and Automation, 2006. ICRA 2006. Proceedings 2006 IEEE International Conference on. IEEE, 2006, pp. $1680-1685$.

[39] W. Kang, S. Nam, Y. Han, and S. Lee, "Improved heading estimation for smartphone-based indoor positioning systems," in Personal Indoor and Mobile Radio Communications (PIMRC), 2012 IEEE 23rd International Symposium on. IEEE, 2012, pp. 2449-2453.

[40] J. Geddes and K. Warwick, "Cloud based global positioning system as a safety monitor for dementia patients," in Cybernetic Intelligent Systems (CIS), 2010 IEEE 9th International Conference on. IEEE, 2010, pp. 1-6.

[41] Y. Chen, J. Liu, A. Jaakkola, J. Hyyppa, L. Chen, H. Hyyppa, T. Jian, and R. Chen, "Knowledge-based indoor positioning based on LiDAR aided multiple sensors system for UGVs," in Position, Location and Navigation Symposium-PLANS 2014, 2014 IEEE/ION. IEEE, 2014, pp. 109-114.

[42] Z. Siddiqui, A. H. Abdullah, M. K. Khan, and A. S. Alghamdi, "Smart environment as a service: Three factor cloud based user authentication for telecare medical information system," Journal of medical systems, vol. 38, no. 1, pp. $1-14,2014$

[43] H.-L. Nguyen, E. Castelli, T.-K. Dao, V.-T. Nguyen, and T.-
T. Pham, "Multimodal combination of GPS, WiFi, RFID and step count for user localization," in Ubiquitous Information Technologies and Applications. Springer, 2014, pp. 675681.

[44] S. Higuera de Frutos and M. Castro, "Using smartphones as a very low-cost tool for road inventories," Transportation Research Part C: Emerging Technologies, vol. 38, pp. 136$145,2014$.

[45] F. Sposaro, J. Danielson, and G. Tyson, "iWander: an android application for dementia patients," in Engineering in Medicine and Biology Society (EMBC), 2010 Annual International Conference of the IEEE. IEEE, 2010, pp. 38753878.

[46] J. Maeso Garcia, F. Jorge Hernandez, B. GarciaZapirain Soto, A. Mendez Zorrilla, B. Fernandez-Ruanova, A. Alday Jurado, and J. Llano Hernaiz, "Teleassistance outdoor system for the elderly based on android devices," in Signal Processing and Information Technology (ISSPIT), 2013 IEEE International Symposium on. IEEE, 2013, pp. $000009-000014$.

[47] D. M. West, "Improving health care through mobile medical devices and sensors," Brookings Institution Policy Report, 2013.

[48] P. Borwankar and G. Kulkarni, "Biological parameter monitoring using android smartphone," 2014.

[49] S. H. Ahmed, A. Sayakkara, G. Kim, and D. Kim, "Selforganized e-health application using IEEE 11703: An experimental approach," Procedia Computer Science, vol. 32, pp. 876-881, 2014.

[50] S. A. Tuncer and A. Alkan, "Abdominal image segmentation on android based mobile devices," in Signal Processing and Communications Applications Conference (SIU), 2014 22nd. IEEE, 2014, pp. 806-809.

[51] E. Sourla, A. Tsakalidis, and G. Tzimas, "Proposing a novel monitoring and early notification system for heart diseases," in Information Technology in Bio-and Medical Informatics. Springer, 2013, pp. 88-102.

[52] M. M. H. Lahr, P. C. A. J. Vroomen, G. J. Luijckx, D. J. Zee, R. Vos, and E. Buskens, "Prehospital factors determining regional variation in thrombolytic therapy in acute ischemic stroke," International Journal of Stroke, 2014.

[53] C. Lambert, S. Vinson, F. Shofer, and J. Brice, "The relationship between knowledge and risk for heart attack and stroke," Journal of Stroke and Cerebrovascular Diseases, vol. 22, no. 7, pp. 996-1001, 2013.

[54] D. Heckerman, A tutorial on learning with Bayesian networks. Springer, 1998. 